\title{
ON HYPERSINGULAR INTEGRALS AND ANISOTROPIC BESSEL POTENTIAL SPACES
}

BY

H. DAPPA AND W. TREBELS

\begin{abstract}
In this paper we characterize anisotropic potential spaces in terms of hypersingular integrals of mixed homogeneity with respect to a general dilation matrix.
\end{abstract}

1. Introduction. The purpose of this paper is to give equivalent characterizations of the anisotropic Bessel potential spaces $\mathcal{L}_{\alpha}^{p}, \alpha>0$ and $1<p<\infty$, in terms of hypersingular integrals. Anisotropic Bessel potential spaces have been earlier introduced by Lizorkin [4] and shown [5] to be equivalent with the $\mathcal{L}_{\alpha^{-}}^{p}$ spaces considered here in the case of diagonal dilation matrices; for special instances of these potential spaces see also Sadosky and Cotlar [8] and Torchinsky [14]. The investigation of hypersingular integrals in the case of the standard Bessel potentials (i.e., isotropic ones) has been carried out by Stein [10] and Wheeden $[15]$, in the case of anisotropic potential spaces with respect to a diagonal dilation matrix by Lizorkin [5]. For related work see $[\mathbf{9}, \mathbf{3}, \mathbf{7}, \mathbf{1}]$. Our methods of proof essentially consist in using Fourier multiplier techniques. To fix ideas let us give some notation. $\mathbf{R}^{n}$ denotes the $n$-dimensional Euclidean space with elements $x, \xi, \ldots$ and scalar product $x \cdot \xi=x \xi=\sum_{j=1}^{n} x_{j} \xi_{j} ; \mathbf{R}_{0}^{n}=\mathbf{R}^{n} \backslash\{0\}$. Let $P$ be a real $n \times n$ matrix whose eigenvalues $\lambda_{j}$ have positive real parts; set $\alpha_{m}=\min _{j=1, \ldots, n} \operatorname{Re} \lambda_{j}, \alpha_{M}=\max _{j=1, \ldots, n} \operatorname{Re} \lambda_{j}$ and as a trace of $P$ set $\nu=\operatorname{tr}(P)$. As in Stein and Wainger [12] associate to $P$ the dilation matrix $A_{t}=t^{P}$ and a distance function $r$, defined by

$$
r(x)=1 / t, \quad B A_{t} x \cdot A_{t} x=1, \quad x \neq 0,
$$

where $B$ is real, positive definite, symmetric and defined by $\left(P^{\prime}\right.$ being the adjoint of $P$ )

$$
B=\int_{0}^{\infty} e^{-t P^{\prime}} e^{-t P} d t
$$

Analogously, the adjoint distance function $\rho$ is defined by

$$
t \rho(x)=1, \quad B^{\#} A_{t}^{\prime} x \cdot A_{t}^{\prime} x=1, \quad x \neq 0, B^{\#}=\int_{0}^{\infty} e^{-t P} e^{-t P^{\prime}} d t .
$$

Then it is shown in [12] that $r, \rho \in C\left(\mathbf{R}^{n}\right)$ are infinitely differentiable for $x \neq 0$. Further, these distance functions satisfy

$$
r\left(A_{t} x\right)=\operatorname{tr}(x), \quad \rho\left(A_{t}^{\prime} \xi\right)=t \rho(\xi)
$$

Received by the editors October 7, 1983 and, in revised form, December 14, 1983.

1980 Mathematics Subject Classification. Primary 42B15, 46E35. 
and for any $\varepsilon>0$ (see e.g. $[\mathbf{1 2}]$ ) one has

$$
\max \{r(x), \rho(x)\} \leq C_{\varepsilon} \begin{cases}|x|^{1 /\left(\alpha_{m}-\varepsilon\right)}, & |x| \rightarrow \infty, \\ |x|^{1 /\left(\alpha_{M}+\varepsilon\right)}, & |x| \rightarrow 0,\end{cases}
$$

and

$$
\min \{r(x), \rho(x)\} \geq C_{\varepsilon} \begin{cases}|x|^{1 /\left(\alpha_{M}+\varepsilon\right)}, & |x| \rightarrow \infty, \\ |x|^{1 /\left(\alpha_{m}-\varepsilon\right)}, & |x| \rightarrow 0 .\end{cases}
$$

(Here and in the following, $C$ will denote constants, in general different from line to line, but always independent of $f$ and $x$.)

On $S\left(\mathbf{R}^{n}\right)$, the space of rapidly decreasing $C^{\infty}$-functions, the Fourier transform $\mathcal{F}$ is defined by

$$
\mathcal{F}(f)(\xi)=\widehat{f}(\xi)=\int f(x) e^{-i \xi x} d x
$$

(where the integration is extended over all of $\mathbf{R}^{n}$ ); by $\mathcal{F}^{-1}$ we denote its inverse, by $\left[L^{1}\left(\mathbf{R}^{n}\right)\right]$ the set of Fourier transforms of all $L^{1}$-functions, and by $M_{p}$ the set of bounded Fourier multipliers on $L^{p}\left(\mathbf{R}^{n}\right)$. We cite from [2] the following useful

LEMMA 1. $m \in B V_{N+1}, N=[n / 2+1]$, implies

$$
\left\|\mathcal{F}^{-1}[m(\rho(\cdot))]\right\|_{1} \leq C\|m\|_{B V_{N+1}} .
$$

Here $B V_{j+1}$ consists of all $C[0, \infty)$-functions vanishing at infinity which are sufficiently smooth and satisfy

$$
\|m\|_{B V_{j+1}}=\int_{0}^{\infty} t^{j}\left|d m^{(j)}(t)\right|<\infty .
$$

Define the anisotropic Bessel potential kernel $G_{\alpha}(x)=G_{\alpha, P^{\prime}}(x)$ by

$$
\widehat{G_{\alpha}}(\xi)=(1+\rho(\xi))^{-\alpha} \text {. }
$$

By Lemma 1 it is clear that $G_{\alpha} \in L^{1}\left(\mathbf{R}^{n}\right)$ for $\alpha>0$. The Bessel potential spaces $\mathcal{L}_{\alpha}^{p}$ (with respect to $P^{\prime}$ ) are now defined as

$$
\mathcal{L}_{\alpha}^{p}=\left\{f \in L^{p}: f=G_{\alpha} * g, g \in L^{p}\right\}, \quad 1 \leq p \leq \infty, \alpha>0,
$$

and normed by $\|f\|_{p, \alpha}=\|g\|_{p}$. Clearly

$$
\|f\|_{p} \leq C\|f\|_{p, \alpha}, \quad 1 \leq p \leq \infty, \alpha>0 .
$$

By $\chi$ denote a $C^{\infty}(\mathbf{R})$-function which equals 0 if $t \leq 1$ and 1 if $t \geq 2$. The $k$ th central difference operator $\Delta_{h}^{k} f$ is defined by

$$
\Delta_{h} f(x)=f(x+h)-f(x-h), \quad \Delta_{h}^{k} f=\Delta_{h}\left(\Delta_{h}^{k-1} f\right) .
$$

Our main results now read as follows.

THEOREM 1. Let $1<p<\infty, \alpha>0$, and $\kappa$ be an even integer greater than $\alpha / \alpha_{m}$. The following norms are equivalent on $\mathcal{L}_{\alpha}^{p}$ :

$$
\|f\|_{p, \alpha}
$$

$$
\|f\|_{p}+\sup _{\varepsilon>0}\left\|D_{\varepsilon}^{\alpha} f\right\|_{p}, \quad D_{\varepsilon}^{\alpha} f=\int_{r(h) \geq \varepsilon} r(h)^{-\alpha-\nu} \Delta_{h}^{\kappa} f d h
$$




$$
\|f\|_{p}+\left\|\sup _{\varepsilon>0}\left|\int \chi\left(\frac{r(h)}{\varepsilon}\right) r(h)^{-\alpha-\nu} \Delta_{h}^{\kappa} f(x) d h\right|\right\|_{p} .
$$

The equivalence of (i) and (ii) in the case of a diagonal dilation matrix $A_{t}$ is already shown in Lizorkin [5], but the methods of proof there do not work in the general case. Apart from this, a careful reading of the proof presented here shows that the special distance function $r$ may be replaced by a continuous, positive definite function $d$ satisfying

$$
d\left(A_{t} \xi\right)=t d(\xi), \quad d \in C^{L}\left(\mathbf{R}_{0}^{n}\right), \quad L>\max \left\{\left(N \alpha_{M}-\alpha\right) / \alpha_{m} ; n\right\} .
$$

Moreover, we may replace $r(h)^{-\alpha-\nu}$ by $\Omega(h) r(h)^{-\alpha-\nu}$, where $\Omega$ is $A_{t}$-homogeneous of degree 0 and belongs to $C^{L}\left(\mathbf{R}_{0}^{n}\right)$. Concerning the forward differences $\dot{\Delta}_{h} f(x)=$ $f(x+h)-f(x)$ and $\dot{\Delta}_{h}^{k} f=\dot{\Delta}_{h}\left(\dot{\Delta}_{h}^{k-1} f\right)$, Lizorkin [5] showed that

$$
\dot{\Delta}_{h} f=\frac{1}{2} \Delta_{h / 2}^{2} f+\frac{1}{2} \Delta_{h} f .
$$

Since $r$ is an even function this implies, for $\kappa>\alpha / \alpha_{m}$,

$$
\begin{aligned}
\int_{r(h) \geq \varepsilon} r(h)^{-\nu-\alpha} \dot{\Delta}_{h}^{\kappa} f(x) d h \\
=2^{-\kappa-1} \sum_{j=0}^{\kappa}\left(\begin{array}{l}
\kappa \\
j
\end{array}\right)\left(1+(-1)^{\kappa+j}\right) \int_{r(h) \geq \varepsilon} r(h)^{-\nu-\alpha} \Delta_{h / 2}^{2 j} \Delta_{h}^{\kappa-j} f(x) d h .
\end{aligned}
$$

Thus, our results also apply for the forward differences.

COROLlaRY 1. If $f \in \mathcal{L}_{\alpha}^{p}$ then, under the hypotheses of Theorem 1,

$$
\int_{r(h) \geq \varepsilon} r(h)^{-\alpha-\nu} \Delta_{h}^{\kappa} f d h
$$

converges in $L^{p}$ for $\varepsilon \rightarrow 0+$ and

$$
\int \chi\left(\frac{r(h)}{\varepsilon}\right) r(h)^{-\alpha-\nu} \Delta_{h}^{\kappa} f(x) d h
$$

converges for almost all $x \in \mathbf{R}^{n}$ as $\varepsilon \rightarrow 0+$.

Part (i) is proved in [5] for diagonal dilation matrices. For the proof of Theorem 1 we need the following technical lemmas.

LEMMA 2. If one defines the function $J(\xi)$ by

$$
J(\xi)=\int \chi(r(h)) r(h)^{-\alpha-\nu} e^{i \xi h} d h,
$$

then $J \in C^{\infty}\left(\mathbf{R}_{0}^{n}\right)$ and $J$ is rapidly decreasing at infinity. In particular, $\chi(\rho(\xi))$ $\times \rho(\xi)^{-\alpha} J(\xi)$ is an $S$-function.

There is an analogous partial result in [5] stating that $\int r(h)^{-\nu-\alpha} \sin ^{k} \xi h d h$ is infinitely differentiable except at the origin. But the method of proof given in $\S 3$ differs from that in [5]. 
Lemma 3. Denote by $D_{\#}^{\gamma} f=\mathcal{F}^{-1}\left[\left(1+|\xi|^{2}\right)^{\gamma / 2} f\right]$ the Bessel derivative of $f \in$ $S^{\prime}$ of order $\gamma>0$. Let $1<q \leq 2$ and $m_{j}(\xi)=m\left(A_{2^{j}}^{\prime} \xi\right)$; assume $D_{\#}^{\gamma} m_{j} \in L_{\text {loc }}^{q}\left(\mathbf{R}_{0}^{n}\right)$ for $\gamma q>n$ with

$$
B V_{q, \gamma}[m]:=\sum_{j \in Z}\left\|D_{\#}^{\gamma}\left(m_{j} \phi\right)\right\|_{q}<\infty
$$

where $\phi \in C^{\infty}\left(\mathbf{R}^{n}\right)$ is a bump function with support contained in $\left\{\xi: \frac{1}{2} \leq \rho(\xi) \leq 2\right\}$. Then $m \in\left[L^{1}\left(\mathbf{R}^{n}\right)\right]$ and, if $q<p \leq \infty$,

$$
\left\|\sup _{t>0}\left|\left(\mathcal{F}^{-1} m\right)_{t} * f\right|\right\|_{p} \leq C B V_{q, \gamma}[m]\|f\|_{p}, \quad f \in L^{p},
$$

where we use the notation $g_{t}(x)=t^{\nu} g\left(A_{t} x\right)$.

We recall that for integer $\beta, 1<p<\infty$, one can identify the classical Bessel potential space with the corresponding Sobolev space (cf. $[11$, p. 135]); hence

$$
\sum_{j \in Z} \sum_{|\sigma| \leq \beta}\left(\int_{1 / 2 \leq \rho(\xi) \leq 2}\left|D^{\sigma} m_{j}(\xi)\right|^{q} d \xi\right)^{1 / q}<\infty
$$

ensures $B V_{q, \gamma}[m]<\infty$ for $\beta \geq \gamma$.

Lemma 3 is a variant of the well-known Bernstein theorem and in some sense an extension insofar as it states that the maximal function generated via convolution with $\left(F^{-1} m\right)_{t}$ is a bounded operator on $L^{p}, 1<q<p \leq \infty$. The same method of proof given in $\S 3$ also yields the following variant.

LEMMA $3^{\prime}$. Let $m \in L_{\text {loc }}^{q}\left(\mathbf{R}_{0}^{n}\right), 1 \leq q \leq 2, A_{t}=\operatorname{diag}\left(t^{\alpha_{1}}, \ldots, t^{\alpha_{n}}\right)$ and

$$
\sum_{j \in Z}\left\|\mathcal{F}^{-1}\left[\prod_{k=1}^{n}\left(1+\xi_{k}^{2}\right)^{\gamma / 2}\right] *\left(m_{j} \phi\right)\right\|_{q}=c_{m, \gamma}<\infty, \quad \gamma q>1 .
$$

Then

$$
\sup _{t>0}\left|\left(\mathcal{F}^{-1} m\right)_{t} * f(x)\right| \leq C C_{m, \gamma}\left(M\left(|f|^{q}\right)(x)\right)^{1 / q}
$$

where $M f$ is the classical Hardy-Littlewood maximal function with respect to rectangles (cf. $[13, p .53])$ having sides parallel to the axes. (2.14).

Another variant of Lemma $3^{\prime}$ with a particularly simple proof will follow in

2. Proof of Theorem 1. (i) and (ii) are equivalent norms. Suppose $f \in \mathcal{L}_{\alpha}^{p} \cap S$; then

$$
\left(D_{\varepsilon}^{\alpha} f\right) \widehat{(\xi)}=\left(I^{-\alpha} f\right) \widehat{(\xi)} \widehat{K^{\prime}}\left(A_{\varepsilon}^{\prime} \xi\right),
$$

where

$$
\begin{aligned}
& \left(I^{-\alpha} f\right) \widehat{(\xi)}=\rho(\xi)^{\alpha} \widehat{f}(\xi), \\
& K^{-}(\xi)=\rho(\xi)^{-\alpha} \int_{\rho(h) \geq 1} k(\xi, h) d h, \quad k(\xi, h)=\left(e^{i h \xi}-e^{-i h \xi}\right)^{\kappa} r(h)^{-\alpha-\nu} .
\end{aligned}
$$


Now decompose $\hat{K^{-}}=\hat{K_{1}}+\hat{K_{2}}+\hat{K_{3}}$, where

$$
\begin{aligned}
& \hat{K_{1}}(\xi)=\chi(\rho(\xi)) \rho(\xi)^{-\alpha} \int_{r(h) \geq 1} k(\xi, h) d h \\
& \hat{K_{2}}(\xi)=-(1-\chi(\rho(\xi))) \rho(\xi)^{-\alpha} \int_{r(h) \leq 1} k(\xi, h) d h, \\
& \widehat{K_{3}}(\xi)=(1-\chi(\rho(\xi))) \rho(\xi)^{-\alpha} \int k(\xi, h) d h .
\end{aligned}
$$

If we can show that

$$
K_{1}, K_{2} \in L^{1}\left(\mathbf{R}^{n}\right), \quad \widehat{K_{3}} \in M_{p}, \quad 1<p<\infty
$$

then it follows from (2.1) that for all $f \in S$ we have

$$
\left\|D_{\varepsilon}^{\alpha} f\right\|_{p} \leq C\left\|I^{-\alpha} f\right\|_{p} \leq C\left\|\mathcal{F}^{-1}\left[(1+\rho(\xi))^{\alpha} f\right]\right\|_{p}=C\|f\|_{p, \alpha}
$$

since $1-t^{\alpha}(1+t)^{-\alpha}$ satisfies the hypotheses of Lemma 1 , and therefore, $\mathcal{F}^{-1}\left[\rho(\xi)^{\alpha}(1+\rho(\xi))^{-\alpha}\right]$ is a bounded measure. Now $S$ is dense in $\mathcal{L}_{\alpha}^{p}$ so that (2.4) holds for all $f \in \mathcal{L}_{\alpha}^{p}$ uniformly in $\varepsilon>0$. This in combination with (1.5) proves that (ii) is a weaker norm than (i) provided we can establish (2.3). Concerning $\widehat{K_{1}}$ first observe that $\widehat{K_{1}}$ is continuous with $\widehat{K_{1}}(0)=0$. Next, since $\chi(\rho(\xi)) \rho(\xi)^{-\alpha} \in\left[L^{1}\left(\mathbf{R}^{n}\right)\right]$ by Lemma 1 , and

$$
\left\|\int_{r(h) \geq 1} r(h)^{-\alpha-\nu} \Delta_{h}^{\kappa} f d h\right\|_{1} \leq C\|f\|_{1}
$$

for all $f \in L^{1}$, one has obviously $K_{1} \in L^{1}\left(\mathbf{R}^{n}\right)$. Concerning $\widehat{K_{2}}$ note that for a sufficiently high difference order $\kappa$ one has $D^{\sigma} K_{2} \in L^{2}\left(\mathbf{R}^{n}\right)$ for all $|\sigma| \leq N=[n / 2]+1$ so that $K_{2} \in L^{1}\left(\mathbf{R}^{n}\right)$ by the Carlson-Beurling inequality. If one is interested in small difference orders $\kappa, \kappa>\alpha / \alpha_{m}$, one can e.g. use the first part of Lemma 3 . Concerning $\widehat{K_{3}}(\xi)=(1-\chi(\rho(\xi))) m(\xi)$, observe that

$$
m(\xi)=\int\left(e^{i h \xi^{\prime}}-e^{-i h \xi^{\prime}}\right)^{\kappa} r(h)^{-\alpha-\nu} d h, \quad \xi^{\prime}=A_{1 / \rho(\xi)}^{\prime} \xi,
$$

shows that $m(\xi) \neq 0$ for all $\xi \neq 0$ ( $\kappa$ is an even integer) and $m$ is $A_{t}^{\prime}$-homogeneous of degree 0 . Hence, by Proposition 4 in [6], we obtain $m \in M_{p}, 1<p<\infty$, once we can prove $D^{\sigma} m \in C\left(\mathbf{R}_{0}^{n}\right)$ for all $|\sigma| \leq N$. Since $\rho(\xi)^{-\alpha} \in C^{\infty}\left(\mathbf{R}_{0}^{n}\right)$, we have to consider

$$
\begin{aligned}
\rho(\xi)^{\alpha} m(\xi)= & \int(1-\chi(r(h))) k(\xi, h) d h \\
& +\sum_{l=0}^{k}\left(\begin{array}{l}
k \\
l
\end{array}\right) \int \chi(r(h)) r(h)^{-\alpha-\nu} e^{i(2 l-k) \xi h} d h
\end{aligned}
$$

The first term as well as the contribution $l=k / 2$ of the second one on the right side are clearly $C^{\infty}$-functions of $\xi$. The remaining ones are of type of Lemma 2 . Thus $m \in M_{p}, 1<p<\infty$, and since $1-\chi(\rho(\xi)) \in S$ we have $K_{3} \in M_{p}$ and (2.3) is proved. 
Conversely, let the expression in (ii) be finite. First we observe that 1$t^{\alpha}\left(1+\chi(t) t^{\alpha}\right)^{-1} \in B V_{N+1}$ and hence, by Lemma 1 and the convolution theorem,

$$
\begin{aligned}
\|f\|_{p, \alpha} & \leq C\left\{\|f\|_{p}+\left\|\mathcal{F}^{-1}\left[\chi(\rho(\xi)) \rho(\xi)^{\alpha} f\right]\right\|_{p}\right\} \\
& \leq C\left\{\|f\|_{p}+\sup _{\varepsilon>0}\left\|\mathcal{F}^{-1}\left[\chi(\rho(\xi)) \rho(\xi)^{\alpha} \hat{\phi}\left(A_{\varepsilon}^{\prime} \xi\right) f\right]\right\|_{p}\right\}
\end{aligned}
$$

for an arbitrary $S$-function $\phi$ with $\widehat{\phi(0)}=1$; here the latter inequality holds since $\phi_{\varepsilon}=\varepsilon^{-\nu} \phi\left(A_{1 / \varepsilon}\right)$ is an approximate identity for $\varepsilon \rightarrow 0+$ and thus, in particular,

$$
\|g\|_{p}=\lim _{\varepsilon \rightarrow 0+}\left\|\phi_{\varepsilon} * g\right\|_{p} \leq \sup _{\varepsilon>0}\left\|\phi_{\varepsilon} * g\right\|_{p}
$$

Now

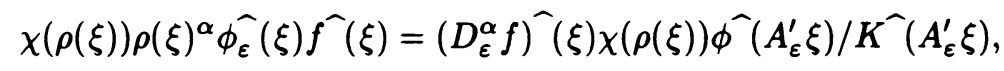

where

$$
\widehat{K^{-}}(\xi)=\rho(\xi)^{-\alpha} \int_{\tau(h) \geq 1} k(\xi, h) d h=m(\xi)-\mu(\xi)
$$

with $m(\xi)$ defined as in (2.5) and

$$
\mu(\xi)=\rho(\xi)^{-\alpha} \int_{r(h) \leq 1} k(\xi, h) d h=\int_{r(h) \leq \rho(\xi)} k\left(\xi^{\prime}, h\right) d h, \quad \xi^{\prime}=A_{1 / \rho(\xi)}^{\prime} \xi .
$$

We now choose $\phi \in S$ in such a way that on the compact support of $\hat{\phi}$ the function $K^{\Upsilon}$ does not vanish. Note that $m(\xi) \neq 0$ for all $\xi \in \mathbf{R}_{0}^{n}, m \in C^{\infty}\left(\mathbf{R}_{0}^{n}\right)$, and is $A_{t}^{\prime}$-homogeneous of degree zero so that

$$
\inf _{\xi \in \mathbf{R}_{\mathbf{0}}^{n}}|m(\xi)|=\inf \left\{\left|m\left(\xi^{\prime}\right)\right|: \rho\left(\xi^{\prime}\right)=1\right\}=\delta>0 .
$$

Since $k\left(\xi^{\prime}, h\right)$ is locally integrable with respect to $h$ it is clear that $\lim _{\xi \rightarrow 0} \mu(\xi)=0$; further, the first representation of $\mu$ shows $\mu \in C^{\infty}\left(\mathbf{R}_{0}^{n}\right)$. Hence choose $\phi \in S$ so that $|\mu(\xi)| \leq \delta / 2$ for all $\xi \in \operatorname{supp} \hat{\phi}$. Then, with $\psi(\xi)=1$ on $\operatorname{supp} \hat{\phi}, \psi \epsilon$ $C^{\infty}\left(\mathbf{R}^{n}\right)$ having appropriate compact support,

$$
\frac{\widehat{\phi}(\xi)}{\widehat{K(\xi)}}=\frac{1}{m(\xi)} \frac{\widehat{\phi(\xi)}}{1-\psi(\xi) \mu(\xi) / m(\xi)}=\frac{M(\xi)}{m(\xi)} .
$$

By Lemma 3 there holds $\psi(\xi) \mu(\xi) / m(\xi) \in\left[L^{1}\left(\mathbf{R}^{n}\right)\right]^{-}$so that by Wiener's theorem $M \in\left[L^{1}\left(\mathbf{R}^{n}\right)\right]^{\wedge}$ and since, by $[6]$, also $1 / m \in M_{p}, 1<p<\infty$, we conclude from (2.7) that

$$
\begin{aligned}
\left\|\mathcal{F}^{-1}\left[\chi(\rho(\xi)) \rho(\xi)^{\alpha} \widehat{\phi_{\varepsilon}} \hat{f}\right]\right\|_{p} & \leq C\left\|\mathcal{F}^{-1}[\chi(\rho(\cdot))] * D_{\varepsilon}^{\alpha} f\right\|_{p} \\
& \leq C \sup _{\varepsilon>0}\left\|D_{\varepsilon}^{\alpha} f\right\|_{p},
\end{aligned}
$$

i.e., in combination with (2.6) the assertion for all $f \in S$ and hence for all $f \in L^{p}$ with finite norm (ii) in Theorem 1.

(i) and (iii) are equivalent norms. First suppose $f \in \mathcal{L}_{\alpha}^{p}$. For $\varepsilon>0$ define on $S$

$$
E_{\varepsilon}^{\alpha} f=\int \chi\left(\frac{r(h)}{\varepsilon}\right) r(h)^{-\alpha-\nu} \Delta_{h}^{\kappa} f d h .
$$


Analogously to (2.1) we have

$$
\left(E_{\varepsilon}^{\alpha} f\right) \widehat{\Upsilon}(\xi)=\left(I^{-\alpha} f\right) \widehat{\Upsilon}(\xi) \widehat{K}\left(A_{\varepsilon}^{\prime} \xi\right)
$$

where this time

$$
\begin{aligned}
& \hat{K^{-}(\xi)}=\rho(\xi)^{-\alpha} \int \chi(r(h)) k(\xi, h) d h=\sum_{j=1}^{3} \hat{K_{j}} \widehat{(\xi)}, \\
& \hat{K_{1}}(\xi)=\chi(\rho(\xi)) \rho(\xi)^{-\alpha} \int \chi(r(h)) k(\xi, h) d h, \\
& \widehat{K_{2}}(\xi)=-(1-\chi(\rho(\xi))) \rho(\xi)^{-\alpha} \int(1-\chi(r(h))) k(\xi, h) d h, \\
& \widehat{K_{3}}(\xi)=(1-\chi(\rho(\xi))) \rho(\xi)^{-\alpha} \int k(\xi, h) d h .
\end{aligned}
$$

By the same methods as above one can show $K_{1}, K_{2} \in L^{1}\left(\mathbf{R}^{n}\right)$ and $\widehat{K_{3}}(\xi)=$

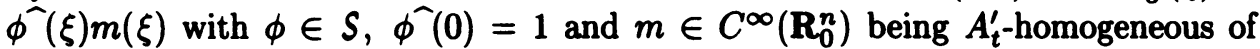
degree 0 . Also, defining $T_{m}$ via $\left(T_{m} \psi\right)^{-}=m(\xi) \hat{\psi}, \psi \in S$, we have

$$
E_{\varepsilon}^{\alpha} f=K_{1, \varepsilon} * I^{-\alpha} f+K_{2, \varepsilon} * I^{-\alpha} f+\phi_{\varepsilon} * T_{m} I^{-\alpha} f .
$$

Since $I^{-\alpha}: \mathcal{L}_{\alpha}^{p} \rightarrow L^{p}$ is continuous, the representation (2.10) holds for all $f \in \mathcal{L}_{\alpha}^{p}$ almost everywhere. In particular,

$$
\begin{aligned}
\sup _{\varepsilon>0}\left|E_{\varepsilon}^{\alpha} f(x)\right| \leq & \sup _{\varepsilon>0}\left|K_{1, \varepsilon} * I^{-\alpha} f(x)\right| \\
& +\sup _{\varepsilon>0}\left|K_{2, \varepsilon} * I^{-\alpha} f(x)\right|+\sup _{\varepsilon>0}\left|\phi_{\varepsilon} * T_{m} I^{-\alpha} f(x)\right| .
\end{aligned}
$$

Clearly, there exists a nonnegative decreasing majorant $L(\xi)=C(1+|\xi|)^{-n-1}$ of $\phi$; then, by [6] the last term is majorized by the maximal function

$$
\mathcal{M g}(x)=\sup _{t>0}\left(L_{t} *|g|\right)(x), \quad g=T_{m} I^{-\alpha} f
$$

and

$$
\left\|\sup _{\varepsilon>0}\left|\phi_{\varepsilon} * T_{m} I^{-\alpha} f(x)\right|\right\|_{p} \leq C\left\|T_{m} I^{-\alpha} f\right\|_{p} \leq C\|f\|_{p, \alpha} .
$$

Concerning $K_{1}$, we note that

$$
\begin{aligned}
\hat{K_{1}}(\xi)= & \chi(\rho(\xi)) \rho(\xi)^{-\alpha}\left(\begin{array}{c}
\kappa \\
\kappa / 2
\end{array}\right)(-1)^{\kappa / 2} \int \chi(r(h)) r(h)^{-\alpha-\nu} d h \\
& +\chi(\rho(\xi)) \rho(\xi)^{-\alpha} \sum_{l \neq \kappa / 2}\left(\begin{array}{c}
\kappa \\
l
\end{array}\right)(-1)^{\kappa-l} \int \chi(r(h)) r(h)^{-\alpha-\nu} e^{i(2 l-\kappa) \xi h} d h .
\end{aligned}
$$

By Lemma 2, the second term on the right side is an $S$-function so that an estimate analogous to (2.13) holds. Concerning the first term, consider first for $\lambda, \Lambda \in N, \lambda=$ $n+2, \Lambda>(n+2) \alpha_{M}-\nu$,

$$
R_{\lambda, \Lambda, t}(x)=\mathcal{F}^{-1}\left[\left(1-\rho(\xi)^{\Lambda} / t\right)_{+}^{\lambda}\right](x) ;
$$


obviously, since $\widehat{R_{\lambda, \Lambda}} \in C^{n+1}\left(\mathbf{R}^{n}\right)$, there holds $R_{\lambda, \Lambda}(x) \leq C(1+|x|)^{-n-1}$, i.e., $R_{\lambda, \Lambda}$ has a radial integrable majorant. Further,

$$
\begin{aligned}
\left|\mathcal{F}^{-1}\left[m\left(\rho(\xi)^{\Lambda}\right)\right] * f(x)\right| & =C\left|\int_{0}^{\infty} R_{\lambda, \Lambda, s} * f(x) s^{\lambda} m^{(\lambda+1)}(s) d s\right| \\
& \leq C\|m\|_{B V_{\lambda+1}} \mathcal{M} f(x)
\end{aligned}
$$

(see (2.12)) by [6]. But certainly $\chi\left(t^{1 / \Lambda}\right) t^{-\alpha / \Lambda} \in B V_{\lambda+1}$ and hence

$$
\sup _{\varepsilon>0}\left|K_{1, \varepsilon} * I^{-\alpha} f(x)\right| \leq C \mathcal{M}\left(I^{-\alpha} f\right)(x) \text {. }
$$

Finally $\widehat{K_{2}}$ satisfies the hypotheses of Lemma 3 which can be seen as follows. $\widehat{K_{2}}$ has compact support so that we have only to show $\sum_{l=-\infty}^{L} B_{l}<\infty$ for some finite $L$, where

$$
B_{l}=\sum_{|\sigma| \leq n}\left(\int_{1 / 2 \leq \rho(\xi) \leq 2}\left|D^{\sigma} \hat{K_{2}}\left(A_{2^{\prime}}^{\prime} \xi\right)\right|^{q} d \xi\right)^{1 / q} .
$$

But this is obvious since for all $\xi, 1 / 2 \leq \rho(\xi) \leq 2$, there holds

$$
\left|D^{\sigma} \widehat{K_{2}}\left(A_{2^{l}}^{\prime} \xi\right)\right| \leq C \min \left\{2^{l\left(\kappa\left(\alpha_{m}-\varepsilon\right)-\alpha\right)}, 2^{l\left(\alpha_{m}-\varepsilon\right)}\right\}, \quad l \in-\mathbf{N}, \sigma \in \mathbf{N}_{0}^{n} .
$$

Summarizing we obtain the desired result by taking $L^{p}$-norms in (2.11) and using the second inequality in (2.4). Conversely, let (iii) be finite. Then

$$
\|f\|_{p, \alpha} \leq C\left\{\|f\|_{p}+\sup _{\varepsilon>0}\left\|E_{\varepsilon}^{\alpha} f\right\|_{p}\right\} \leq C\left\{\|f\|_{p}+\left\|\sup _{\varepsilon>0}\left|E_{\varepsilon}^{\alpha} f\right|\right\|_{p}\right\},
$$

where the first inequality is analogous to the proof of (ii) $\rightarrow$ (i) and the second one is obvious. Thus all is proved.

3. Proof of Corollary 1. (i) By (2.1) and (2.2) we have

$$
D_{\varepsilon}^{\alpha} f=\sum_{i=1}^{3} \mathcal{F}^{-1}\left[\widehat{K_{i}}\left(A_{\varepsilon}^{\prime} \xi\right)\right] * I^{-\alpha} f
$$

where $I^{-\alpha} f \in L^{p}$ since $f \in \mathcal{L}_{\alpha}^{p}$ (see (2.4)). By the properties of $K_{1}$ and $K_{2}$ discussed in $\S 2$ it is clear that

$$
\lim _{\varepsilon \rightarrow 0+}\left\|K_{i, \varepsilon} * I^{-\alpha} f\right\|_{p}=0, \quad i=1,2
$$

(cf. $\left[13\right.$, p. 11]). We recall $m\left(A_{\varepsilon}^{\prime} \xi\right)=m(\xi)$ and, therefore,

$$
\widehat{K_{3}}\left(A_{\varepsilon}^{\prime} \xi\right)=\left(1-\chi\left(\rho\left(A_{\varepsilon}^{\prime} \xi\right)\right)\right) m(\xi) .
$$

Since $\mathcal{F}^{-1}\left(1-\chi\left(\rho\left(A_{\varepsilon}^{\prime} \xi\right)\right)\right)$ is an approximate identity for $\varepsilon \rightarrow 0+$ it is clear that

$$
\mathcal{F}^{-1}\left[\widehat{K_{3}}\left(A_{\varepsilon}^{\prime} \xi\right)\right] * I^{-\alpha} f \rightarrow T_{m} I^{-\alpha} f
$$

in $L^{p}$ for $\varepsilon \rightarrow 0+$, i.e., the assertion.

(ii) This is an immediate consequence of Theorem 1 in combination with Theorem 3.12 , Chapter II in $[\mathbf{1 3}]$, since by $(2.10)$ we have for $f \in S$ with $0 \notin \operatorname{supp} f^{-}$that

$$
\lim _{\varepsilon \rightarrow 0+} K_{i, \varepsilon} * I^{-\alpha} f(x)=0, \quad i=1,2
$$


and

$$
\lim _{\varepsilon \rightarrow 0} \mathcal{F}^{-1}\left(1-\chi\left(\rho\left(A_{\varepsilon}^{\prime} \xi\right)\right)\right) * T_{m} I^{-\alpha} f(x)=T_{m} I^{-\alpha} f(x) \quad \text { a.e. }
$$

Proof OF LEMMA 2. By definition it is clear that $J$ is continuous on $\mathbf{R}^{n}$ and vanishes at infinity by the Riemann-Lebesgue lemma. Let $\xi \neq 0$, in particular $\xi_{j} \neq 0$; denote by $\xi^{(j)}=\left(\xi_{1}, \ldots, \xi_{j-1}, \xi_{j+1}, \ldots, \xi_{n}\right) \in \mathbf{R}^{n-1}$; let $\sigma \in \mathbf{N}_{0}^{n}$ be arbitrary, $|\sigma|=l$, choose $L>\left(l \alpha_{M}-\alpha\right) / \alpha_{m}$, and integrate partially $L$ times with respect to $h_{j}$ :

$$
\begin{aligned}
J(\xi)= & \int_{\mathbf{R}^{n-1}} e^{i \xi^{(j)} h^{(j)}} \int_{-\infty}^{\infty} e^{i \xi_{j} h_{j}} \chi(r(h)) r(h)^{-\alpha-\nu} d h_{j} d h^{(j)} \\
= & \frac{1}{\left(i \xi_{j}\right)^{L}} \int_{\mathbf{R}^{n}} e^{i \xi h}\left(\frac{\partial}{\partial h_{j}}\right)^{L}\left\{\chi(r(h)) r(h)^{-\alpha-\nu}\right\} d h \\
= & \frac{1}{\left(i \xi_{j}\right)^{L}} \sum_{l=0}^{L-1}\left(\begin{array}{l}
L \\
l
\end{array}\right) \int_{\mathbf{R}^{n}} e^{i \xi h}\left(\frac{\partial}{\partial h_{j}}\right)^{l} r(h)^{-\alpha-\nu}\left(\frac{\partial}{\partial h_{j}}\right)^{L-l} \chi(r(h)) d h \\
& +\frac{1}{\left(i \xi_{j}\right)^{L}} \int_{\mathbf{R}^{n}} e^{i \xi h} \chi(r(h))\left(\frac{\partial}{\partial h_{j}}\right)^{L} r(h)^{-\alpha-\nu} d h \\
= & J_{1}(\xi)+J_{2}(\xi) .
\end{aligned}
$$

In the case $l<L$ the function $\left(\partial / \partial h_{j}\right)^{L-l} \chi(r(h))$ has compact support away from the origin; hence $J_{1} \in C^{\infty}\left(\mathbf{R}_{0}^{n}\right)$. Since

$$
\left|\left(\frac{\partial}{\partial h_{j}}\right)^{L} r(h)^{-\alpha-\nu}\right| \leq C_{\varepsilon} r(h)^{-\alpha-\nu-L\left(\alpha_{m}-\varepsilon\right)}, \quad|h| \rightarrow \infty,
$$

and since for $\sigma^{\prime} \in \mathbf{N}_{0}^{n},\left|\sigma^{\prime}\right| \leq l$, there holds $\left|h^{\sigma^{\prime}}\right| \leq|h|^{l} \leq C_{\varepsilon} r(h)^{l\left(\alpha_{M}+\varepsilon\right)}$ for $|h| \rightarrow \infty$, we have

$$
\begin{aligned}
D^{\sigma} J_{2}(\xi) & =\sum_{\sigma=\sigma^{\prime}+\sigma^{\prime \prime}} D^{\sigma^{\prime \prime}}\left(\frac{1}{i \xi_{j}}\right)^{L} D^{\sigma^{\prime}} \int_{\mathbf{R}^{n}} e^{i \xi h} \chi(r(h))\left(\frac{\partial}{\partial h_{j}}\right)^{L} r(h)^{-\alpha-\nu} d h \\
& \leq C \sum_{\sigma=\sigma^{\prime}+\sigma^{\prime \prime}}\left|D^{\sigma^{\prime \prime}}\left(\frac{1}{i \xi_{j}}\right)^{L}\right| \int_{\mathbf{R}^{n}}\left|h^{\sigma^{\prime}}\right| \chi(r(h))\left|\left(\frac{\partial}{\partial h_{j}}\right)^{L} r(h)^{-\alpha-\nu}\right| d h \\
& \leq C \sum_{\sigma=\sigma^{\prime}+\sigma^{\prime \prime}}\left|D^{\sigma^{\prime \prime}}\left(\frac{1}{i \xi_{j}}\right)^{L}\right| \int_{r(h) \geq 1} r(h)^{-\alpha-\nu-L \alpha_{m}+l \alpha_{M}+(L+l) \varepsilon} d h
\end{aligned}
$$

which for small $\varepsilon>0$ converges because $l \alpha_{M}-\alpha-L \alpha_{m}<0$ by our choice of $L$. It is clear that by integrating partially $k L$ times we may produce an arbitrary decrease in $\xi_{j}$ at infinity. Since $\xi_{j}$ and the order of differentiation, namely $l$, was arbitrary, there holds

$$
\sum_{j=1}^{n} \xi_{j}^{L} D^{\sigma} J(\xi)=O(1), \quad|\xi| \rightarrow \infty,
$$

i.e. $J \in C^{\infty}\left(\mathbf{R}_{0}^{n}\right)$ and $J$ is rapidly decreasing. Since $\chi(\rho(\xi)) \rho(\xi)^{-\alpha} \in C^{\infty}\left(\mathbf{R}^{n}\right)$ with support away from the origin is only slowly increasing, the last assertion of Lemma 2 is obvious. 
ProOF OF LEMMA 3. Madych proved in [6, Theorem 5] that $B V_{2, \beta}[m]<\infty$ for $\beta>n / 2$ is sufficient for $m$ to belong to $\left[L^{1}\left(\mathbf{R}^{n}\right)\right]^{-}$. If now $\gamma>n / q$ then we can find $\beta>n / 2$ such that $\gamma-n / q \geq \beta-n / 2$. Hence, by $[11$, pp. 119 and 133], $B V_{2, \beta}[m] \leq C B V_{q, \gamma}[m]$ and thus $m \in\left[L^{1}\left(\mathbf{R}^{n}\right)\right]^{\wedge}$. Let $\varphi \in C^{\infty}\left(\mathbf{R}^{1}\right)$ with $\operatorname{supp} \varphi \subset\left[\frac{1}{2}, 2\right]$ and $\sum_{j \in Z} \varphi\left(2^{-j} t\right)=1$ for $t>0$; set $\varphi\left(2^{-j} \rho(\xi)\right)=\phi_{j}(\xi), \phi_{0}=\phi$. Since $m \in\left[L^{1}\left(\mathbf{R}^{n}\right)\right]^{-}$we have, for all $f \in L^{p}$,

$$
\left|\left(\mathcal{F}^{-1} m\right)_{t} * f(x)\right| \leq \sum_{j \in Z}\left|\left(\mathcal{F}^{-1}\left[m \phi_{j}\right]\right)_{t} * f(x)\right|=\sum_{j \in Z} I_{j}(x) .
$$

Now observe that $\mathcal{F}^{-1}\left[m \phi_{j}\right](x)=2^{j \nu} \mathcal{F}^{-1}\left[m_{j} \phi\right]\left(A_{2}, x\right)$ and apply Hölder's inequality to obtain

$$
\begin{aligned}
I_{j}(x) \leq & \left\{\left(2^{j} t\right)^{\nu} \int\left|\left(1+\left|A_{2^{j} t}(x-y)\right|^{2}\right)^{\gamma / 2} \mathcal{F}^{-1}\left[m_{j} \phi\right]\left(A_{2^{j} t}(x-y)\right)\right|^{q^{\prime}} d y\right\}^{1 / q^{\prime}} \\
& \cdot\left\{\left(2^{j} t\right)^{\nu} \int\left(1+\left|A_{2^{j} t}(x-y)\right|^{2}\right)^{-\gamma q / 2}|f(y)|^{q} d y\right\}^{1 / q} \\
= & \left\|\left(1+|\cdot|^{2}\right)^{\gamma / 2} \xi^{-1}\left[m_{j} \phi\right]\right\|_{q^{\prime}}\left\{K_{2^{j} t} *|f|^{q}(x)\right\}^{1 / q},
\end{aligned}
$$

where $K(x)=\left(1+|x|^{2}\right)^{-\gamma q / 2}$. It is easy to verify that $K$ and $A_{t}$ satisfy the assumptions of Theorem 3 in [6]; thus, the maximal operator

$$
\mathcal{M} g(x)=\sup _{t>0}\left|K_{t} * g(x)\right|
$$

is bounded from $L^{p}$ into itself if $1<p \leq \infty$. Using this and the Hausdorff-Young inequality we conclude from (3.1) and (3.2), if $q<p \leq \infty$, that

$$
\left\|\sup _{t>0}\left|\left(\xi^{-1} m\right)_{t} * f\right|\right\|_{p} \leq \sum_{j \in Z}\left\|D_{\#}^{\gamma}\left(m_{j} \phi\right)\right\|_{q}\left\|\mathcal{M}\left(|f|^{q}\right)\right\|_{p / q}^{1 / q} \leq C B V_{q, \gamma}[m]\|f\|_{p}
$$

\section{REFERENCES}

1. S. Chanillo, Hypersingular integrals and parabolic potentials, Trans. Amer. Math. Soc. 267 (1981), 531-547.

2. H. Dappa and W. Trebels, On $L^{1}$-criteria for quasi-radial Fourier multipliers with applications to some anisotropic function spaces, Anal. Math. 9 (1983), 275-289.

3. J. Horváth, Composition of hypersingular integral operators, Applicable Anal. 7 (1977/78), 171190.

4. P. I. Lizorkin, Nonisotropic Bessel potentials, imbedding theorems for Sobolev spaces $L_{p}^{\left(r_{1}, \ldots, r_{n}\right)}$ with fractional derivatives, Soviet Math. Dokl. 7 (1966), 1222-1226.

5. _ Description of the spaces $L_{p}^{r}\left(\mathbf{R}^{n}\right)$ in terms of singular difference integrals, Math. USSR-Sb. 10 (1970), 77-89.

6. W. Madych, On Littlewood-Paley functions, Studia Math. 50 (1974), 43-63.

7. V. A. Nogin, Inversion of the generalized Riesz potentials with the symbols that degenerate binearty on a hyperplane, Math. Notes 32 (1982), 637-642.

8. C. Sadosky and M. Cotlar, On quasi-homogeneous Bessel potential operators, Proc. Sympos. Pure Math., vol. 10, Amer. Math. Soc., Providence, R.I., 1967, pp. 275-287.

9. S. G. Samko, Generalized Riesz potentials and hypersingular integrals, their symbols and inversion, Soviet Math. Dokl. 18 (1977), 97-101.

10. E. M. Stein, The characterization of functions arising as potentials. I, Bull. Amer. Math. Soc. 67 (1961), 102-104. 
11. Singular integrals and diffenentiabitity properties of functions, Princeton Univ. Press, Princeton, N.J., 1970.

12. E. M. Stein and S. Wainger, Problems in harmonic analysis related to the curvature, Bull. Amer. Math. Soc. 84 (1978), $1239-1295$.

13. E. M. Stein and G. Weiss, Introduction to Fourier anahysis on Euctidean spaces, Princeton Univ. Press, Princeton, N.J., 1971.

14. A. Torchinsky, On spaces of Riesz potentials, preprint, Cornell University.

15. R. L. Wheeden, On hypersingular integrals and Lebesgue spaces of differentiable functions. I, II, Trans. Amer. Math. Soc. 134 (1968), 421-436; 139 (1969), 37-53.

Fachbereich Mathematik, TH DaRmstadt, Schlossgartenstrasse 7, D-6100 DARMSTADT, FEDERAL REPUBLIC OF GERMANY 